\title{
Sensitivity of forecast errors to initial and lateral boundary conditions
}

\author{
By NILS GUSTAFSSON ${ }^{1 *}$, ERLAND KÄLLÉN ${ }^{1,3}$ and SIGURDUR THORSTEINSSON ${ }^{1}{ }^{1}$ Swedish \\ Meteorological and Hydrological Institute, S-60176 Norrköping, Sweden; ${ }^{2}$ Icelandic Meteorological Office, \\ Bústadavegi 9, IS-150 Reykjavík, Iceland; ${ }^{3}$ Stockholm University, Department of Meteorology, Arrhenius \\ Laboratory, S-10691 Stockholm, Sweden
}

(Manuscript received 30 July 1997; in final form 18 November 1997)

\begin{abstract}
The adjoint of a limited area model has been used to study the sensitivity of $12 \mathrm{~h}$ forecast errors to initial and lateral boundary conditions. Upper troposphere potential vorticity and mean sea level pressure verification scores for 1 month of operational forecasts from the Swedish Meteorological and Hydrological Institute were used to select 2 cases with particularly poor forecast performance. The sensitivity experiments show that errors in initial data is the most likely explanation for one of the forecast failures, while errors in initial as well as lateral boundary data can explain the 2 nd forecast failure. Results from the sensitivity experiments with respect to the lateral boundary conditions indicate that poor quality lateral boundary conditions may be improved by utilizing subsequent downstream observations within the model integration area. This result is of great significance with regard to the possibilities for applying 4-dimensional variational data assimilation (4DVAR) for limited area forecast models. Results from the sensitivity experiments also reveal, however, that the lateral boundary treatment in operational limited area models needs to be improved with regard to the mathematical formulation. It is furthermore shown that modifications to be applied to the lateral boundary conditions need to be determined with appropriate time resolution and that some filtering of these lateral boundary modifications has to be introduced to avoid enhanced high-frequency gravity wave noise in the vicinity of the lateral boundaries.
\end{abstract}

\section{Introduction}

There are a number of possible causes for errors in short-range numerical weather forecasts based on limited area models. Considering the predominance of advective and baroclinic processes on the time-scales over the few days of short-range forecasts, initial and lateral boundary conditions become the most obvious candidates. With regard to errors in the initial data, a problem that limitedarea models share with global forecast models, it has been demonstrated that many forecast failures may be related to difficulties in analysing baroclinic

\footnotetext{
* Corresponding author
}

structures over data-sparse areas like the Atlantic Ocean and the Pacific (Källén and Huang, 1988; Gustafsson et al., 1997). The adjoint model technique (Le Dimet and Talagrand, 1986) has proven to be a powerful tool to relate the origin of numerical forecast errors to errors in the initial data through sensitivity experiments (Rabier et al., 1996; Gustafsson and Huang, 1996). The idea behind such studies is to identify a case with large forecast errors and then to trace the errors back to the initial state by calculating the gradient of the forecast error norm with respect to the initial conditions. For this purpose, the adjoint model is utilized to project the gradient of the forecast error norm from the forecast time back to the initial data 
time. Subtracting a fraction of the gradient of the forecast error norm from the initial condition and performing a new (sensitivity) forecast run with changed initial conditions should then lead to an improved forecast, as verified by the same forecast error norm.

For limited area models, poor lateral boundary conditions is another important error source distinct from the initial state errors. The specification of lateral boundary conditions is a weak point of limited area model forecasting. Forecast errors may be due to the coarse time and space resolution of the boundary values. The boundary data may be based on an old and thus inaccurate forecast due to delays in the production and dissemination of these data. In addition, an ill-posed mathematical formulation of the boundary condition may also create errors. Such weaknesses in the formulation of the lateral boundary conditions may also contribute to an amplification of forecast errors attributed to parameterisation errors or any other model formulation errors or inaccuracies. In addition to the direct negative effect of poor lateral boundary data during the forecast model integration, there may also be an accumulated negative effect during data assimilation forecast cycles, in particular if the lateral boundaries are situated upstream of data-sparse areas within the model integration area (Gustafsson, 1990).

A widely used measure of the forecast error is the root mean square (rms) and mean (bias) of the difference between forecast fields and observations. Any numerical forecast model will show a growth in time of the rms error averaged over a large forecast sample and a bias growth or decay. From a large forecast sample it is, however, difficult to assess the relative importance of the error sources listed above and thus to determine which parts of the model to improve.

In the present study, we will first examine a time sequence of potential vorticity $(\mathrm{PV})$ and mean sea level pressure $\left(p_{\text {msl }}\right)$ verification scores to isolate cases with exceptionally large forecast errors. From the PV and $p_{\mathrm{msl}}$ error characteristics we will make hypotheses regarding the source of the forecast error. Experience with PV and $p_{\mathrm{msl}}$ verifications in the HIRLAM project (a co-operative limited area model development project between nine European weather services, see Källén, 1995) suggests that the area and ensemble mean $p_{\mathrm{msl}}$ forecast errors are associated with inconsistencies between the limited area model and the model generating the lateral boundary conditions. Area and ensemble average rms forecast errors in the $p_{\mathrm{msl}}$ and the upper air PV, on the other hand, are in general associated with errors in the specification of the initial field. Furthermore, this experience suggests that peaks in the day to day rms forecast error that drift in time with the forecast length depend on inaccuracies in the initial and boundary values, while the errors that remain stationary in time may depend on inconsistencies between forecasts and observations at the time of comparison.

Once we have used the forecast error verification scores to identify poor forecast cases, we will carry out sensitivity experiments with the adjoint model technique to identify the origin of the forecast errors. Previous studies have mainly concentrated on errors in the initial data. Errico et al. (1993) studied the sensitivity of forecast errors with respect to both initial and lateral boundary conditions. A novel feature of the present study is to investigate the sensitivity to initial and lateral boundary data as well as to carry out sensitivity forecast runs using the same model setup. We show how the two sensitivities may be separated and how they can be combined to fully explain a case with a significant forecast error which appears to originate in the vicinity of a lateral boundary. The formulation of the boundary sensitivity experiment is discussed in some detail; in particular we point out principal difficulties in applying the adjoint technique to a limited area model with an ill-posed boundary condition. We also discuss time filters needed to avoid a dominant gravity wave component in the perturbations created by the modified lateral boundary values in the sensitivity experiment.

We have mainly examined the sensitivity of the 12-h forecast errors to errors in the initial data and to errors in lateral boundary data, provided by a coarser global model. Two forecast situations from February 1995 with poor verification scores were selected. It turned out that one of the cases was dominated by errors in the initial data, while the second case was influenced by errors in initial as well as lateral boundary data. This paper first presents, in Section 2, verification scores gathered over one month. A brief description of the design of the sensitivity experiments is given in Section 3 . Section 4 discusses the results of the sensitivity experiments for the two selected cases. Conclusions are drawn in Section 5. 


\section{Verifications and diagnostics}

The quality of a forecast is measured by comparing the forecast with the actual analysed situation. This is accomplished by an area integration (summation) of rms error and bias deviation from the analysis. A comparison is made on the pressure level grid itself, i.e., for a given level the rms error and bias are calculated as

$\sigma_{\mathrm{rms}}=\sqrt{\frac{1}{K} \sum_{k=1}^{K}\left(S_{k}^{\text {forecast }}-S_{k}^{\text {analysis }}\right)^{2}}$,

$\bar{\sigma}=\frac{1}{k} \sum_{k=1}^{K}\left(S_{k}^{\text {forecast }}-S_{k}^{\text {analysis }}\right)$,

where $\sigma_{\text {rms }}$ is the rms error, $\bar{\sigma}$ is the mean error (bias), $S_{k}^{\text {forecast }}$ and $S_{k}^{\text {analysis }}$ are the gridpoint values of the field under concern at one time level, and $K$ is the number of gridpoints. All gridpoints are given the same weight, i.e., the area they represent is not taken into account.

Once the rms error and bias have been calculated at a pressure level, a bias diagram or bias profile may be drawn.

A diagnostic evaluation of the upper air potential vorticity (PV) and mean sea level pressure $\left(p_{\mathrm{msl}}\right)$ field verification results for the month of February 1995 for the HIRLAM model (Källén, 1995) used in operational runs at SMHI (resolution $0.5^{\circ}, 162 \times 142$ horizontal points and 16 levels) has been carried out. The verification score is computed for the entire integration area.

The rms errors of the $+6 \mathrm{~h}$ forecasts of 06 and 18 UTC initialised upper air PV data are much larger than those of the 00 and 12 UTC initialised upper air PV data due to the fact that new upper air information comes in mainly at 00 and 12 UTC. Therefore the 00 and 12 UTC initialised data are used here.

Fig. 1 shows the average rms-error and bias for February 1995 for $300-500 \mathrm{hPa}$ potential vorticity (solid line) and $p_{\mathrm{msl}}$ (dotted line). The variation with the forecast length shows that:

(i) the rms-error of the $p_{\text {msl }}$ field increases linearly with forecast length;

(ii) the rms-error of the $300-500 \mathrm{hPa}$ potential vorticity also increases with forecast length, indicating errors in the specification of the initial field, but the rate of increase diminishes somewhat for long forecast times;

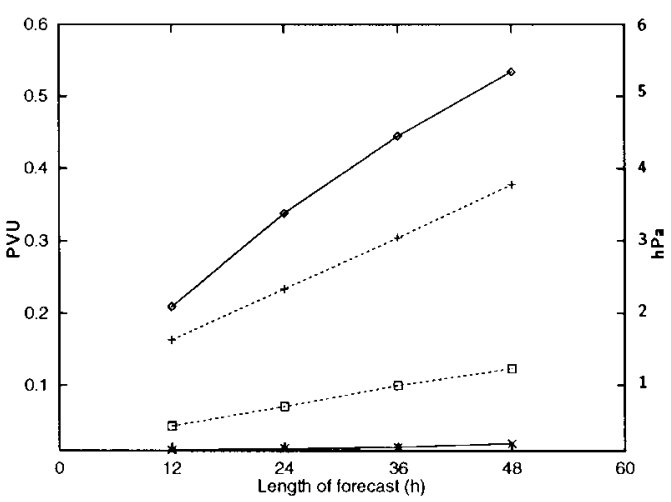

Fig. 1. The average rms error and bias for February 1995 for $300-500 \mathrm{hPa}$ potential vorticity (in PVU, solid line) and $p_{\mathrm{msl}}$ (in $\mathrm{hPa}$, dotted line) from operational forecasts at SMHI. The verification is based on field comparisons. The upper two curves apply to the rms error and the lower two apply to the bias.

(iii) the bias in the 300-500 hPa potential vorticity is small, thus indicating that the internal thermal and wind structures are well maintained;

(iv) the bias in the $p_{\mathrm{msl}}$ increases the entire time; a possible explanation may be an inconsistency between the lateral boundary values and the inner model solution causing an artificial mass flux over the lateral boundaries.

In order to find a case for the further analysis of the nature of the forecast errors, we need to look into the verification scores of the individual forecasts. Fig. 2 shows the time series of the areaaveraged rms error and bias for February of the

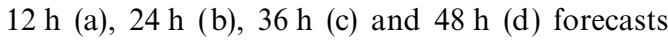
for $300-500 \mathrm{hPa}$ potential vorticity (solid lines), and $p_{\mathrm{msl}}$ (dotted lines) fields in the HIRLAM model. The forecasts were initialised at 00 and 12 UTC. The upper two curves apply to rms error and the lower two apply to the bias.

It is seen from Fig. 2 that there is a high correlation between the rms errors of the 300 $500 \mathrm{hPa}$ potential vorticity and msl pressure fields. The high correlation indicates that there are errors in the specification of the initial or boundary fields for the model. In contrast, the corresponding correlation in bias appears to be less pronounced.

Furthermore, Fig. 2 shows that in some cases peaks in the rms error drift in time at a rate similar to the increase in forecast time, indicating 

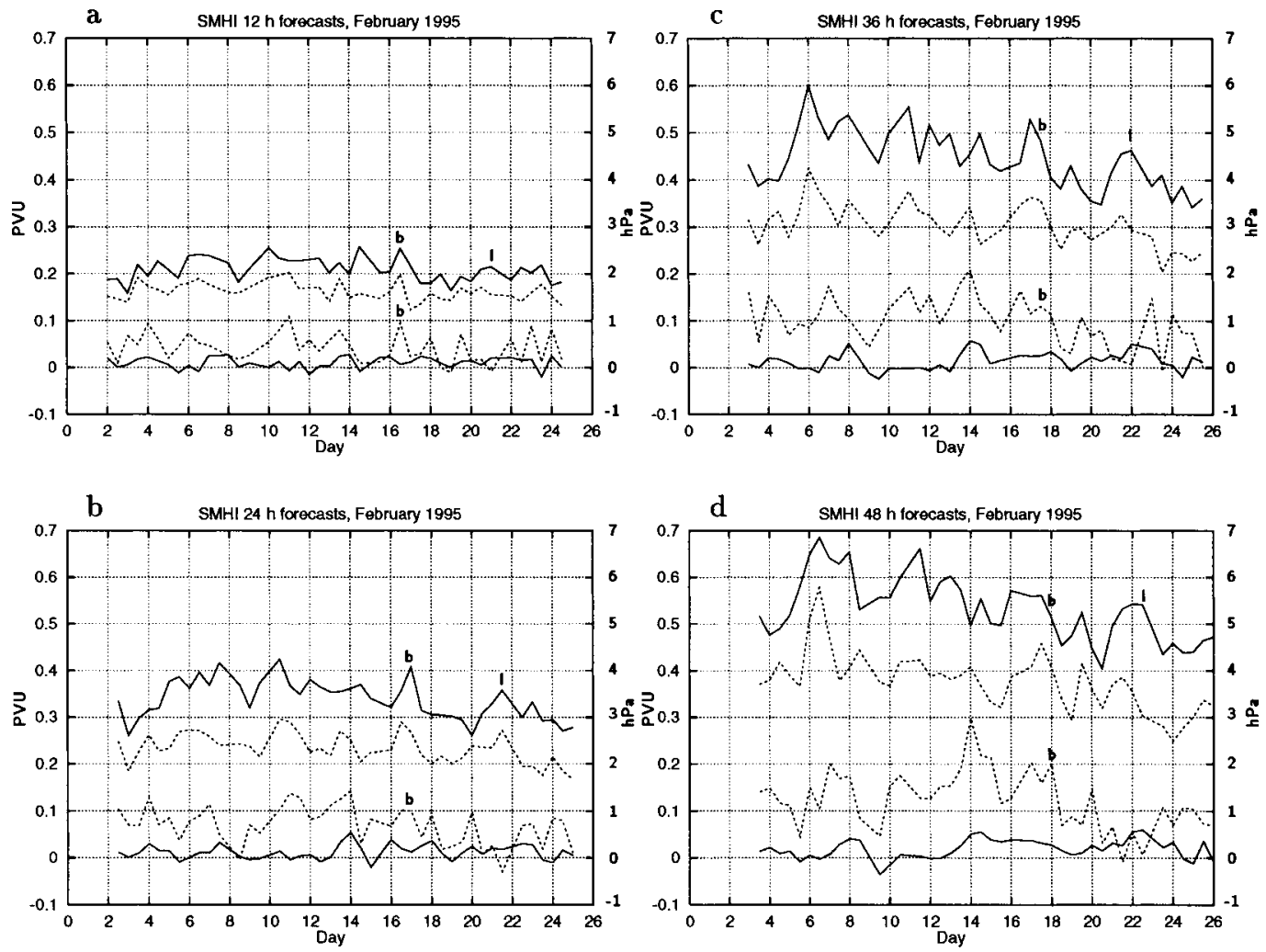

Fig. 2. Time series of the area-averaged rms error and bias for February 1995 of the $12 \mathrm{~h}, 24 \mathrm{~h}, 36 \mathrm{~h}$ and $48 \mathrm{~h}$ forecasts for $300-500 \mathrm{hPa}$ potential vorticity (in PVU, solid line) and $p_{\mathrm{msl}}$ (in $\mathrm{hPa}$, dotted line) fields from the HIRLAM model at SMHI, verified by the associated HIRLAM analysis at SMHI. The upper two curves apply to the rms error and the lower two apply to the bias.

that these errors are due to inaccuracies in the initial data or in the lateral boundary data. In other cases the peaks remain stationary, indicating that the errors were only diagnosed by observations made at the time of comparison and a possible explanation may be a model error which is fixed in space and only occurs in a specific flow situation. However, another possible, and perhaps more probable, explanation for a stationary error is an erroneous observation.

\section{Design of sensitivity experiments}

\subsection{Sensitivity with respect to initial conditions}

To distinguish between errors caused by erroneous boundary conditions and inaccuracies in the initial state, we will carry out sensitivity experiments using the adjoint of the HIRLAM forecast model. We will use the same experimentation technique as described by Gustafsson and Huang (1996). The basic idea of such sensitivity experiments is to carry an adjoint model integration backward in time, starting from forecast errors as given by differences between the verifying analysis and the forecast for a given forecast range. This adjoint backward integration provides us with an estimate of the gradient of a quadratic cost function of the forecast errors with respect to the initial conditions. By subtraction of a fraction of this gradient from the original initial data fields, it is possible to derive an alternative initial state that should result in an improved forecast.

The following energy-related quadratic costfunction $J$ was used in our experiments to measure 
the differences between the verifying analysis and the forecast:

$$
\begin{aligned}
J= & \frac{1}{2} \sum_{x} \sum_{y} \sum_{p}\left[(\Delta u)^{2}+(\Delta v)^{2}+R_{\mathrm{d}} T_{\mathrm{r}}\left(\Delta \ln p_{\mathrm{s}}\right)^{2}\right. \\
& \left.+\frac{C_{\mathrm{p}}}{T_{\mathrm{r}}}(\Delta T)^{2}\right] .
\end{aligned}
$$

The summation in this cost function is taken over all the horizontal and vertical gridpoints after spectral truncation with the same weight for all gridpoints. The differences between analysed and forecast values of the wind components $\Delta u, \Delta v$, $\operatorname{logarithms}$ of surface pressure $\Delta \ln p_{\mathrm{s}}$ and temperature $\Delta T$ are included in $J$. The gas constant for dry air is $R_{\mathrm{d}}, C_{\mathrm{p}}$ is the specific heat at constant pressure for dry air, and $T_{\mathrm{r}}$ is a reference temperature.

A sensitivity experiment over an $L_{\text {win }}$ hour assimilation window is carried out through the following steps.

(1) A non-linear HIRLAM forecast is run, including horizontal diffusion and all physical parameterisation schemes ("reference" forecast run) from initial data valid at year YY, month MM, day DD and hour HH. An adiabatic non-linear normal mode initialisation is applied at the start of the model integration.

(2) The difference between a verifying analysis, valid at YYMMDDHH $+L_{\text {win }}$ hour, and the $L_{\text {win }}$ hour HIRLAM forecast is used to calculate the gradient of the quadratic forecast error cost function $J$, see above, with respect to the forecast model variables at YYMMDDHH $+L_{\text {win }}$ hour.

(3) A backward projection of the gradient field with the adiabatic adjoint HIRLAM, including horizontal diffusion from $+L_{\text {win }}$ hour to +00 hour in order to obtain the gradient of the quadratic forecast error cost function with respect to the initial conditions. The adjoint of the adiabatic non-linear normal mode initialisation is applied at the end of the adjoint model integration.

(4) The gradient of the forecast error cost function with respect to the initial conditions, together with the definition of this cost function, is used to estimate the error of the initial analysis fields. Then, "improved" initial conditions are obtained by subtracting a fraction $(\alpha)$ of these estimated errors of analysis from the original initial condition fields.

(5) A new non-linear HIRLAM forecast is run, including horizontal diffusion and all physical parameterisation schemes, from the modified initial conditions ("sensitivity" forecast run). An adiabatic non-linear normal mode initialisation is applied at the start of the model integration.

In the experiments described below, the scaling factor $\alpha$ was chosen to be 0.1 with regard to the 12-h assimilation window. This scaling factor was chosen by experimentation with different scaling factors.

The forecast model used for the sensitivity experiment is the spectral version of HIRLAM described in Gustafsson (1991) and in Gustafsson and McDonald (1996).

\subsection{Sensitivity with respect to lateral boundary conditions}

Limited-area model forecasting is not only affected by errors of the initial conditions; errors in lateral boundary conditions may also seriously affect the quality of the limited area model forecasts (Gustafsson, 1990). Gustafsson and Huang (1996) avoided the lateral boundary condition problem by using analysis boundaries for their sensitivity experiments. From a technical point of view, it is straightforward to extend the idea of the adjoint model and sensitivity experiments to the treatment of the lateral boundaries (Errico et al., 1993). The spectral HIRLAM uses the Davies (1983) lateral boundary relaxation scheme with a cosine-shaped boundary relaxation factor:

$\psi(t, \boldsymbol{x})=\varepsilon(r(\boldsymbol{x})) \psi^{\mathrm{B}}(t, \boldsymbol{x})+(1 .-\varepsilon(r(\boldsymbol{x}))) \psi^{\mathrm{I}}(t, \boldsymbol{x})$

$\varepsilon(r(\boldsymbol{x}))=0.5\left(1 .+\cos \left(\pi \frac{r(\boldsymbol{x})}{r_{\max }}\right)\right)$,

where $r(x)$ is the distance between the gridpoint $\boldsymbol{x}$ and the lateral boundary, $r_{\max }$ the width of the lateral boundary relaxation zone, $\psi^{\mathrm{B}}$ is the externally provided boundary value, $\psi^{\mathbf{I}}$ the solution of the model equations in the inner integration area and $\psi$ the "boundary relaxed" solution. 
This boundary relaxation is a simple linear weighting, and thus the tangent linear equations for relaxation of lateral boundary perturbations are identical to eqs (4) and (5). The adjoint boundary relaxation expressions are then easily derived:

$\delta \psi_{\mathrm{AD}}^{\mathrm{B}}(t, \boldsymbol{x})=\varepsilon(r(\boldsymbol{x})) \delta \psi_{\mathrm{AD}}(t, \boldsymbol{x})$,

$\delta \psi_{\mathrm{AD}}^{\mathrm{I}}(t, \boldsymbol{x})=(1 .-\varepsilon(r(\boldsymbol{x}))) \delta \psi_{\mathrm{AD}}(t, \boldsymbol{x})$.

Even if the mathematical formulation is straightforward, it is not obvious how to proceed with the practical implementation of the adjoint of the lateral boundary relaxation scheme. In principle, the equation above will give one adjoint boundary perturbation $\delta \psi_{\mathrm{AD}}^{\mathrm{B}}(t, \boldsymbol{x})$, representing a gradient of the forecast error norm with respect to the lateral boundary condition, for each timestep of the model integration. These gradients can be used to create modifications to the original lateral boundary conditions, in analogy with the corresponding modifications to the initial conditions. A technical drawback of this solution is that it requires storing of the adjoint boundary values from all time-steps in the computer. In our case new boundary values are provided only every $6 \mathrm{~h}$, boundary values at intermediate times are calculated through linear interpolation. An alternative is therefore to include also the adjoint of the linear time-interpolation of the boundary values. This has the effect that a boundary perturbation will be available every 6th hour only.

Some preliminary sensitivity experiments with regard to errors in the lateral boundary conditions indicate that it is advantageous to apply the first version of the adjoint of the boundary relaxation scheme, resulting in lateral boundary perturbations to be applied for each timestep of the forward model integration. The spatial structures introduced by the lateral boundary relaxation appeared to become more accurate with perturbations available for every timestep, and this can be understood from the non-linear time variations of the lateral boundary perturbations over a 6-h period. Such non-linear variations in time over a 6-h period cannot be described with linear interpolations from 6 hourly data only. On the other hand, it is also known that the boundary relaxation scheme, as it is utilized for HIRLAM, creates artificial divergent winds and related gravity wave oscillations in the boundary relaxation zone. In order to avoid enhanced gravity wave oscillations in the sensitivity forecast runs, a simple time filter (time averaging) was applied to the lateral boundary perturbations obtained by the adjoint model integration. Experimentation (see subsection 4.2) indicated that time averaging over $\pm 1 \mathrm{~h}$ gave satisfactory results.

A basic weakness of the lateral boundary relaxation technique was revealed during the experimentation with the adjoint of the scheme. The strongest sensitivity of the forecast errors to the lateral boundary conditions occurred in a region of strong physical inflow with a low pressure system passing the lateral boundaries during the time period of the sensitivity experiment. During the forward model integration, the lateral boundary relaxation scheme managed quite well in this inflow region to introduce the time variation of the meteorological fields as given by the coarser resolution model providing the lateral boundary conditions. During the adjoint model backward integration, however, this region of physical inflow becomes an area of outflow of forecast error gradient information. In the present construction of the HIRLAM boundary relaxation scheme both inflow and outflow points are handled identically. Davies (1983) has shown that inflow of information is handled reasonably well at the same time as outflow information is reasonably well absorbed by the boundary zone damping. To achieve this twofold goal, information introduced only at the outermost boundary points will be significantly damped before it reaches the interior of the domain. To introduce inflow information a forcing in the entire boundary zone is required and it can be demonstrated (see Section 7) that most of the information which reaches the interior domain actually comes from grid-points in the middle of the boundary zone. The outermost point is well isolated from the interior domain in order to avoid spurious reflection of outwardly propagating information.

In our sensitivity experiments, we see clearly that the gradient of the forecast error norm with respect to the boundary conditions will be near zero at the outermost boundary zone points. On the other hand, immediately inside the lateral boundary, within the boundary relaxation zone, the calculated boundary perturbation may well achieve a significant magnitude. Such discontinuities of a perturbation introduced in the lateral 
boundary relaxation zone will contribute to an enhanced generation of gravity wave noise. Thus we may find that boundary zone perturbations give rise not only to geostrophically well-balanced perturbations which propagate into the interior domain, but also to undesired gravity waves which are quickly damped out by horizontal diffusion and the semi-implicit time stepping scheme. This result shows that the lateral boundary relaxation scheme needs to be modified to take the occurrence of inflow and outflow regions into account (Orlanski, 1976; Eliassen and Thorsteinsson, 1984; Thorsteinsson, 1988).

\section{Results from sensitivity experiments}

We have carried out two experiments to study the sensitivity of forecast errors to the specification of the initial as well as the lateral boundary data. Both experiments were run with the spectral version of HIRLAM, with a shortest resolved wavelength of approximately $180 \mathrm{~km}$ and with 16 vertical hybrid levels. Both experiments were started from operational SMHI HIRLAM analysis fields, valid $12 \mathrm{~h}$ before the verification time. For the lateral boundary conditions, ECMWF forecast fields based on initial data $24 \mathrm{~h}$ before the verification time, were utilized. The frequency for updating of the lateral boundary conditions was once every $6 \mathrm{~h}$. Linear interpolation was used for the time-interpolation of the lateral boundary data. For calculation of the quadratic forecast error norm and its gradient at the verification time, operational SMHI HIRLAM analysis fields were used.

\subsection{An initial data error case}

The first case study (case marked I in the verification scores of Fig. 2) pertains to a misforecast of rapid surface cyclogenesis south of Greenland and Iceland between 1200 UTC on 20 February and 0000 UTC on 22 February 1995. Fig. 3 shows the operational SMHI HIRLAM analysis of mean sea level pressure and $300 \mathrm{hPa}$ height, respectively, valid at 0000 UTC on 22 February 1995. The low pressure system with a centre pressure of about $970 \mathrm{hPa}$ south of Iceland and the associated baroclinically tilting upper level trough dominate the fields.

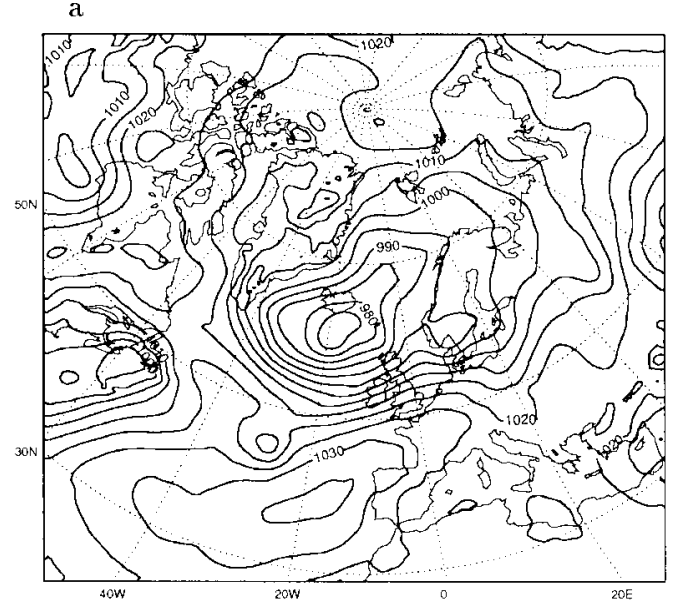

b

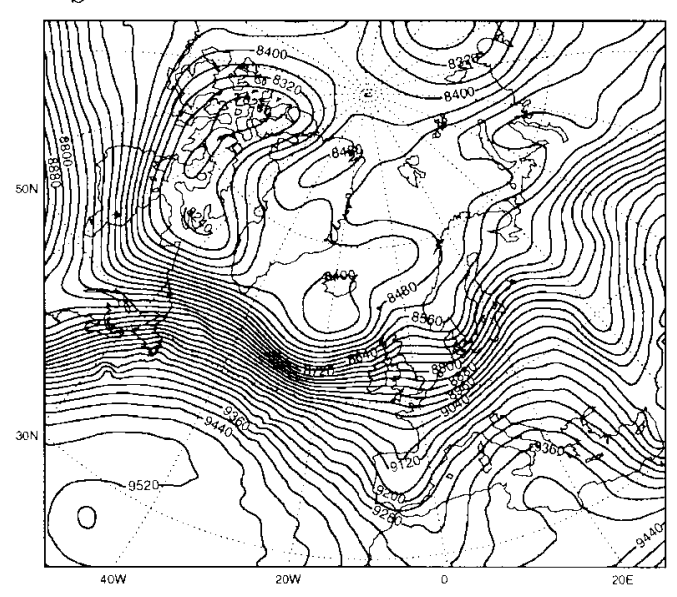

Fig. 3. Operational SMHI analysis for (a) $p_{\mathrm{msl}}$ and (b) $300 \mathrm{hPa}$ height valid at 0000 UTC on 22 February 1995.

The $300 \mathrm{hPa}$ height errors (verification analysis - forecast from the operational SMHI HIRLAM) starting from initial data valid at 1200 UTC on 20 February 1995 have been plotted in Figs. $4 \mathrm{a}-\mathrm{c}$ for the forecast lengths of $+12,+24$ and $+36 \mathrm{~h}$, respectively. Note the significant underestimate in the forecast depth of the upper air trough over the northwest Atlantic southeast of Greenland at $+12 \mathrm{~h}$.

A sensitivity experiment was carried out for the period between 1200 UTC on 20 February and 0000 UTC on 21 February 1995. The differences between the $+12 \mathrm{~h}$ forecast and the corresponding verification analysis, as illustrated in Fig. $4 \mathrm{a}$ at 

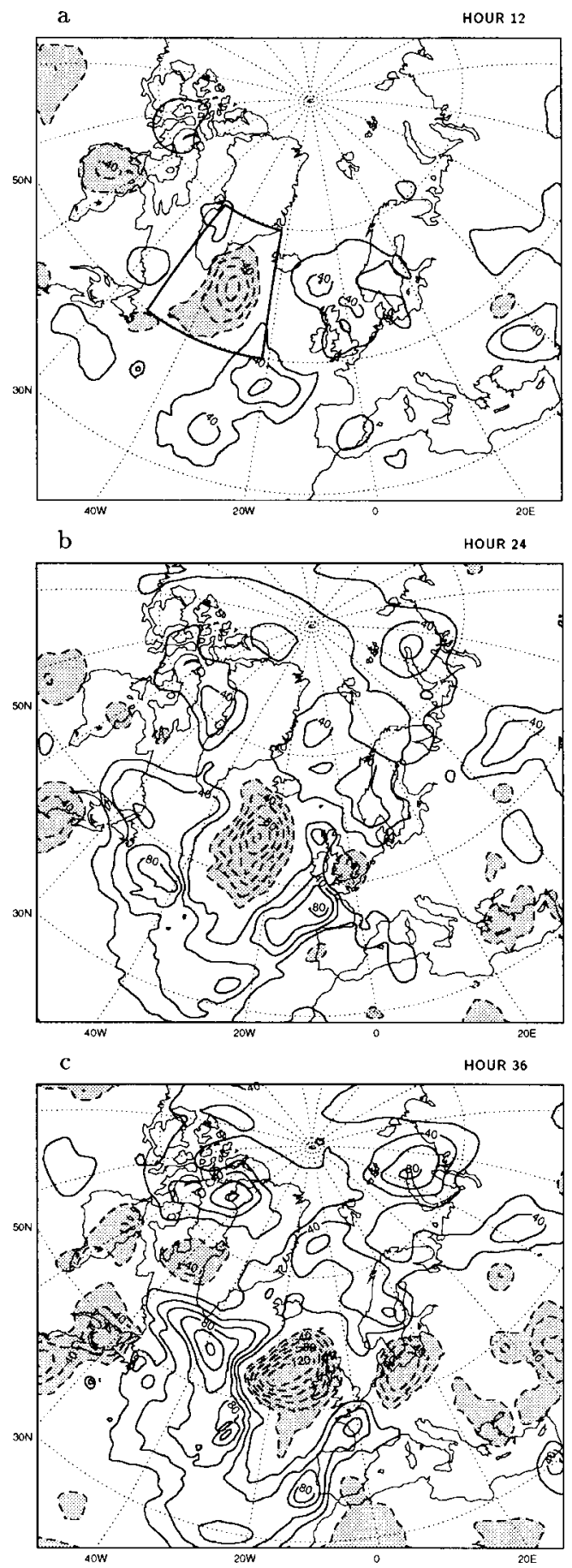

$300 \mathrm{hPa}$, were thus used to provide the adjoint model integration with an input gradient of a forecast error norm. In a first sensitivity experiment the forecast error norm was applied over the complete model integration area. The results of this sensitivity experiment, taking sensitivity to initial data into account only, are illustrated in Fig. 5, which shows the differences between the sensitivity and reference $+0 \mathrm{~h},+6 \mathrm{~h}$ and $+12 \mathrm{~h}$ forecasts for the $300 \mathrm{hPa}$ height. The sensitivity experiment manages to retrieve the main structure and amplitude of the $+12 \mathrm{~h} 300 \mathrm{hPa}$ height error southeast of Greenland. The $+0 \mathrm{~h}$ forecast differences, i.e., the scaled gradient of the forecast error norm at the initial time, include significant sensitivity patterns upstream of this forecast error, southwest of Greenland. Significant sensitivity patterns are present in other areas as well, for example southwest of Ireland and over Newfoundland.

As indicated, the forecast error norm was calculated in the complete model integration area for the first sensitivity experiment. This had the effect of also retrieving forecast errors other than those of main concern for this study. Note, for example, the differences between the $+12 \mathrm{~h}$ sensitivity and reference forecasts southwest of Ireland in Fig. 5c. In order to isolate the origin of the forecast error for the cyclone evolution southeast of Greenland, an area-concentrated forecast error norm was used instead of the full area forecast error norm. First, we subjectively selected the error anomaly at $+12 \mathrm{~h}$ that was believed to be of greatest importance for the detection of the initial forecast error over the NW Atlantic. Then the input to the forecast error norm was put equal to zero outside a subarea, as indicated in Fig. 4a, that includes this error anomaly. The results of the application of this area-concentrated error norm sensitivity experiment were successful (Fig. 6). The retrieved forecast error pattern now includes only the forecast error of main concern in the evolution of

Fig. 4. $300 \mathrm{hPa}$ height error fields (verification analysis - forecast from operational SMHI HIRLAM) for forecast lengths of $12 \mathrm{~h}, 24 \mathrm{~h}$ and $36 \mathrm{~h}$ from 1200 UTC on 20 February 1995. Positive (negative) values are represented by solid (dashed) contours. Contours every $20 \mathrm{~m}$. The shaded areas correspond to errors $\leqslant-20 \mathrm{~m}$. The sector-formed area in (a) is used for calculation of the area-concentrated forecast error norm at $12 \mathrm{~h}$. 


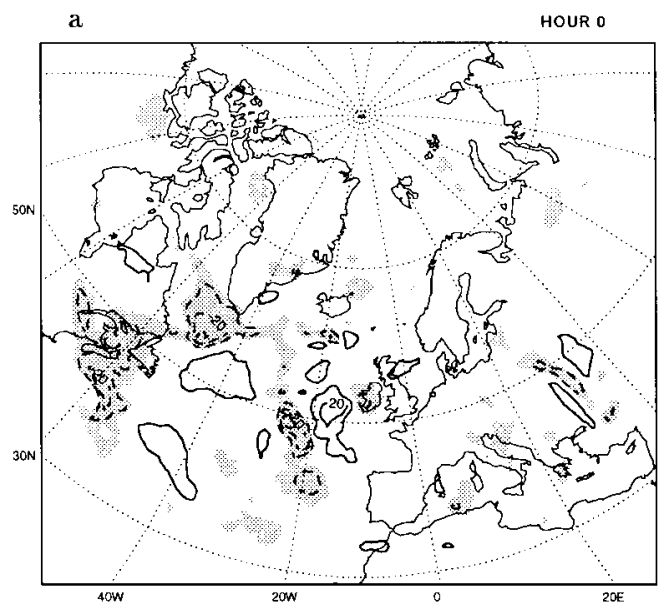

b
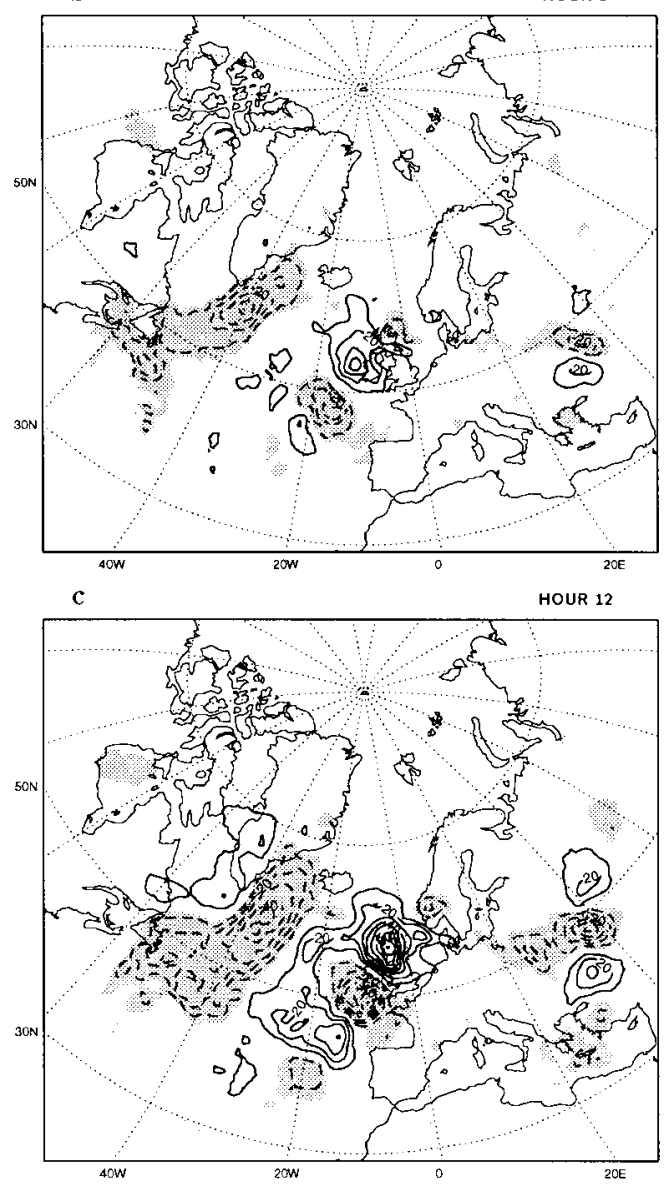

Fig. 5. $300 \mathrm{hPa}$ height differences between the sensitivity and reference for $0 \mathrm{~h}, 6 \mathrm{~h}$ and $12 \mathrm{~h}$ forecasts from 1200 UTC on 20 February 1995 using initial perturbations only with $\alpha=0.1$. The forecast error norm was calculated for the complete model integration area. Positive (negative) values are represented by solid (dashed) contours. Contours every $10 \mathrm{~m}$. The shaded areas correspond to

retrieved forecast errors $\leqslant-20 \mathrm{~m}$.

the error for this study. We may conclude that a main cause of this particular forecast error is an error of the analysis south-west of Greenland, as indicated by the differences between the $+0 \mathrm{~h}$ sensitivity and reference forecasts, i.e., the scaled gradient of the forecast error norm at the initial time, in Fig. 6 a

The signal from this experiment testing the sensitivity of forecast errors with respect to initial data errors was very clear. We cannot exclude, wer, a sensitivity also with respect to lateral boundary errors. Therefore, an experiment to test the sensitivity to initial as well as lateral boundary d was carried out. The procedure for deriving the lateral boundary perturbations, described in section 3.2 and discussed in more detail below for the second case study, was applied with a scaling coefficient $\alpha_{\text {bound }}=5.0$ and with a time averaging period of $\pm 1 \mathrm{~h}$ for the lateral boundary perturbations. Also this experiment was carried out with the area-concentrated forecast error norm, as was used in the previous experiment. The additional impact of the derived lateral boundary perturbations was very marginal, as can be seen by comparing the retrieved forecast error patterns in Fig. 7 with those of the previous experiment in Fig. 6. Another experiment, to test the sensitivity of the forecast errors to perturbations in lateral boundary conditions only, was also carried out. This experiment confirmed what was noticed in the mixed initial and lateral boundary data experiment, that the sensitivity of the forecast error to the lateral boundary conditions was very marginal for this particular forecast error case and this particular area-restricted forecast error norm.

In summary, the adjoint model technique has been shown to give a reasonable result for one individual case, that of 1200 UTC on 20 February 1995, which confirmed the sensitivity of the $12 \mathrm{~h}$ forecast error over the NW Atlantic with respect to initial conditions. 

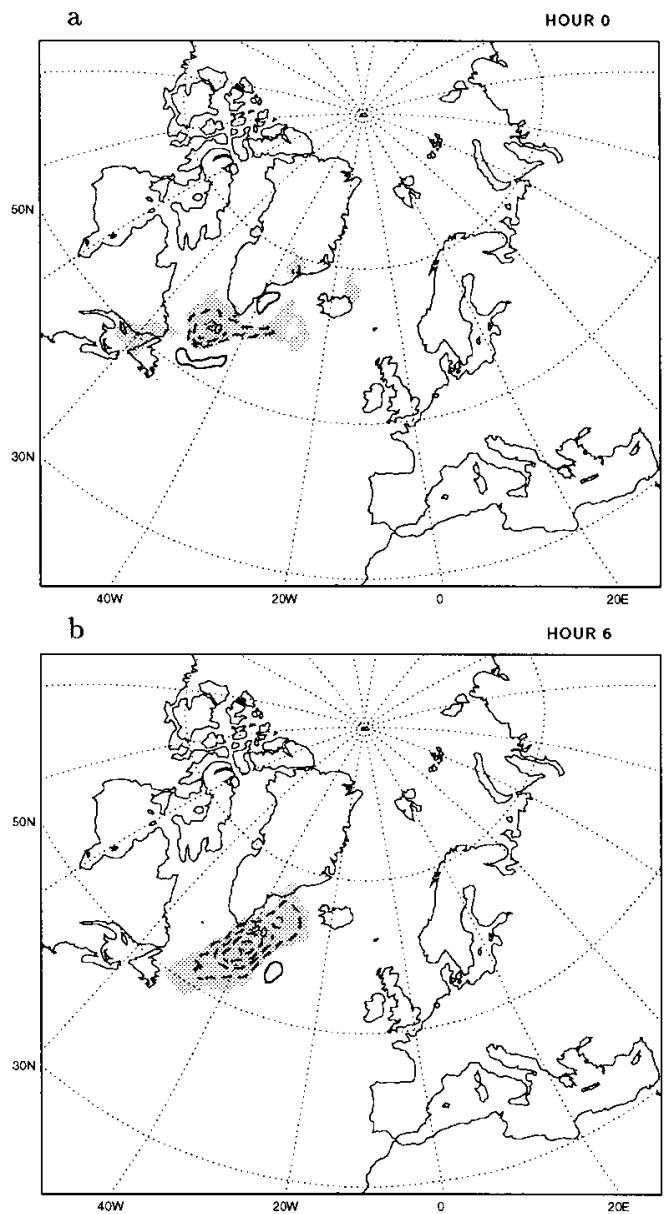

c

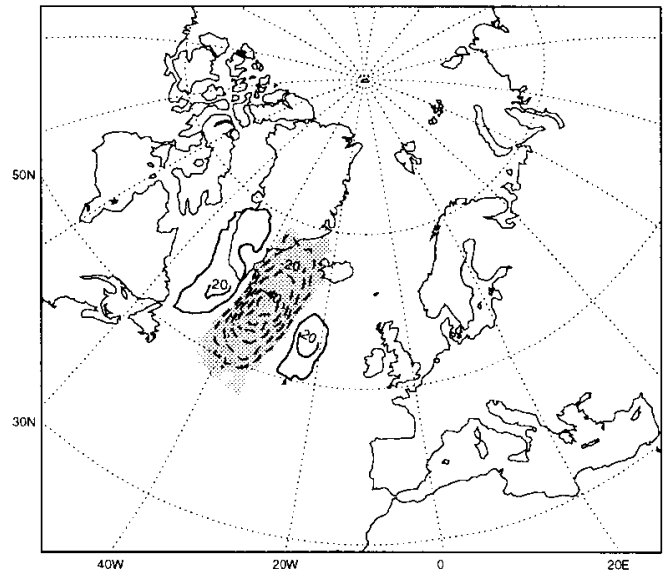

Fig. 6. As in Fig. 5, but based on the area-concentrated $12 \mathrm{~h}$ forecast error norm (Fig. 4a) in the sensitivity forecast run.

\subsection{A mixed initial and lateral boundary data error case}

The case marked (b) in Fig. 2 (occurring on 16 February 1995) has been investigated to determine the impact of initial as well as lateral boundary errors. This case turned out to be particularly sensitive to lateral boundary conditions over eastern Canada, i.e., in an area with good data coverage. Fig. 8 shows the operational SMHI HIRLAM analysis of $p_{\mathrm{msl}}$ and $300 \mathrm{hPa}$ height, respectively, valid at 0000 UTC on 16 February 1995. These fields reveal the low pressure system with a centre msl pressure of about $998 \mathrm{hPa}$ at the west side boundary over eastern Canada.

The $300 \mathrm{hPa}$ height errors (verification analysis - forecast from the operational SMHI HIRLAM) starting from initial data valid at 0000 UTC on 16 February 1995, have been plotted in Figs. 9a, b, c, for forecast lengths of 12, 24 and $36 \mathrm{~h}$, respectively. Note that the operational forecast grossly underestimates the forecast depth of the low propagating through and from the west side boundary over Newfoundland and later over the NW Atlantic. This indicates that the forecast error is likely to be related to poorly described structures in the lateral boundary conditions provided by the coarser resolution global model.

Sensitivity experiments were carried out for the period between 0000 and 1200 UTC on 16 February 1995. Since the purpose of this investigation is to detect the origin of the forecast error over Newfoundland, the area-concentrated forecast error norm at $+12 \mathrm{~h}$, indicated in Fig. 9a by heavy lines, was used.

The first sensitivity experiment was carried out using only the initial data perturbation following the procedure described in Subsection 3.1 with $\alpha=0.1$. The results are shown in Fig. 10 for $300 \mathrm{hPa}$ height and wind differences between the sensitivity and reference $+0 \mathrm{~h},+6 \mathrm{~h}$ and $+12 \mathrm{~h}$ forecasts, respectively. Comparing Figs. 9a and $10 \mathrm{c}$, it is obvious that the $+12 \mathrm{~h}$ forecast error is only partly retrieved by the initial data sensitivity experiment. Considering that the initial perturbation, introduced by the aid of the adjoint model integration, at the initial time of the forecast is placed very close to the lateral boundaries, we may presume that errors in the lateral boundary conditions are also needed for a full explanation of the $+12 \mathrm{~h}$ forecast errors experienced. 

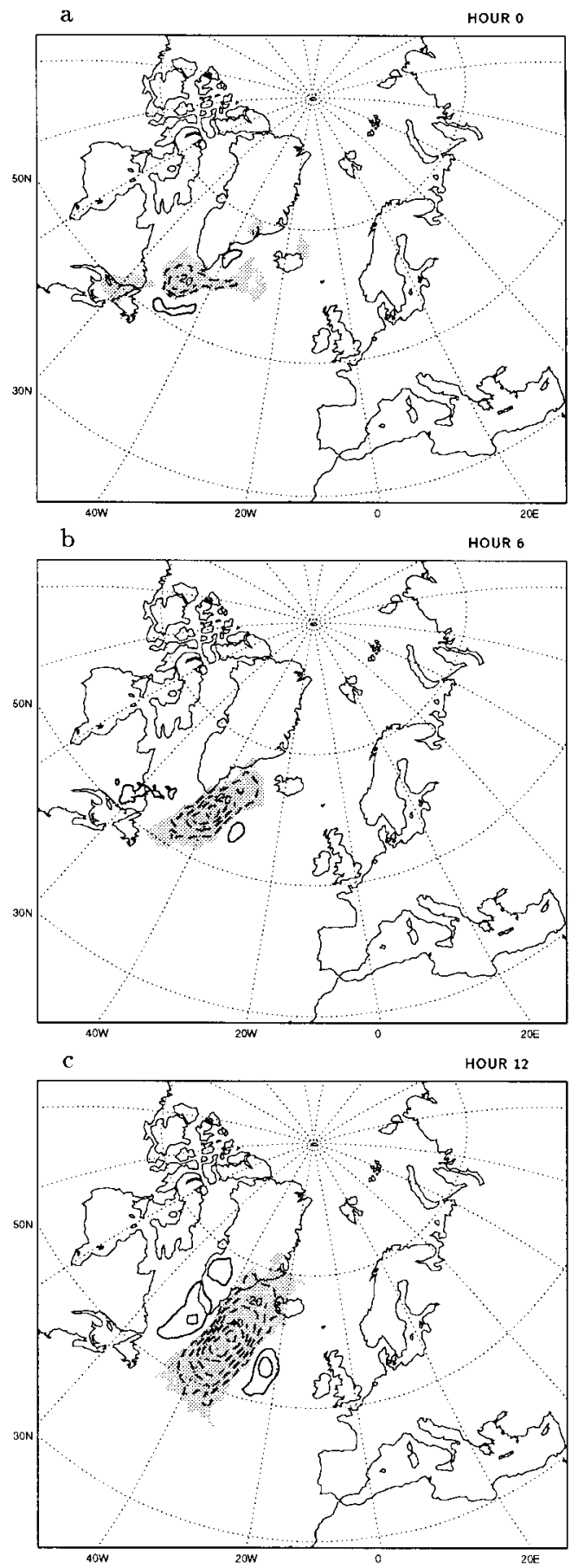

Fig. 7. $300 \mathrm{hPa}$ height differences between the sensitivity and reference for $0 \mathrm{~h}, 6 \mathrm{~h}$ and $12 \mathrm{~h}$ forecasts from 1200 UTC on 20 February 1995 using initial perturbations with $\alpha=0.1$ and boundary perturbations with $\alpha_{\text {bound }}=$ 5.0. The area-concentrated $12 \mathrm{~h}$ forecast error norm (see Fig. 4a) was used in the sensitivity forecast run. Positive (negative) values are represented by solid (dashed) contours. Contours every $10 \mathrm{~m}$. The shaded areas correspond to retrieved forecast errors $\leqslant-20 \mathrm{~m}$.
The second sensitivity experiment was carried out using only the lateral boundary perturbations for the sensitivity forecast run. The procedure for deriving lateral boundary perturbations for each time-step, described in Subsection 3.2, was applied with a time averaging period of $\pm 1 \mathrm{~h}$. In addition, it turned out to be necessary to utilize a scaling coefficient $\alpha_{\text {bound }}=5.0$ for the lateral boundary perturbations in order to achieve the successful results of this sensitivity experiment, as illustrated in Fig. $11 \mathrm{~b}-\mathrm{c}$ for the +6 and $+12 \mathrm{~h}$ forecasts. The needed magnitude of this scaling coefficient may partly be justified by the shorter time-period over which the lateral boundary perturbations in the average are allowed to grow, as compared to the initial data perturbations. More important, however, is that the adjoint boundary relaxation expressions described in Subsection 3.2 also efficiently act as a down-scaling of the gradient of the forecast error norm with respect to the lateral boundary conditions.

Since the initial data perturbation is zero for this particular forecast sensitivity run, we have included the lateral boundary perturbation, i.e., the scaled gradient of the forecast error norm with respect to the lateral boundary conditions, at time $+0 \mathrm{~h}$ in Fig. 11a. We may notice that non-zero values of the lateral boundary perturbations are restricted to the interior of the boundary relaxaAppendix A. We may also notice a significant boundary perturbation in the area of strong inflow upstream of the $+12 \mathrm{~h}$ forecast error area of interest for this case study. It should be pointed (he whole time-series of boundence the sensitivity forecast run in this particular forecast model setup. initial data and the lateral boundary data sensitivtion zone, as explained by the simple analysis in

Comparing the sensitivity difference fields of the 


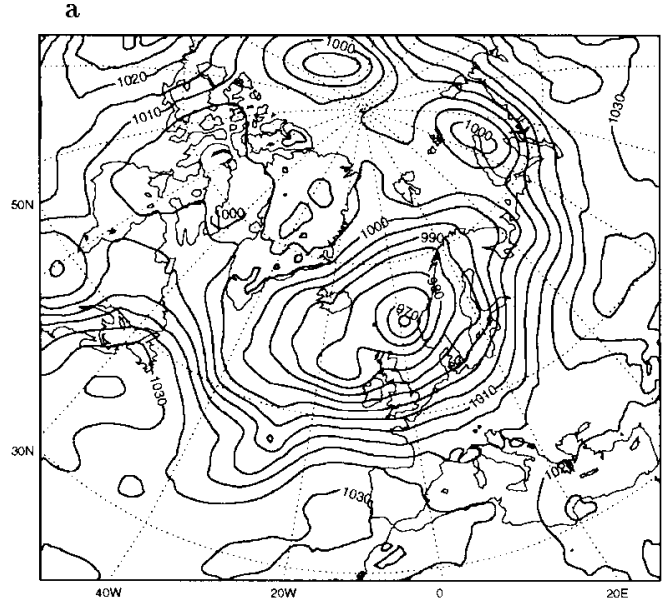

$\mathrm{b}$

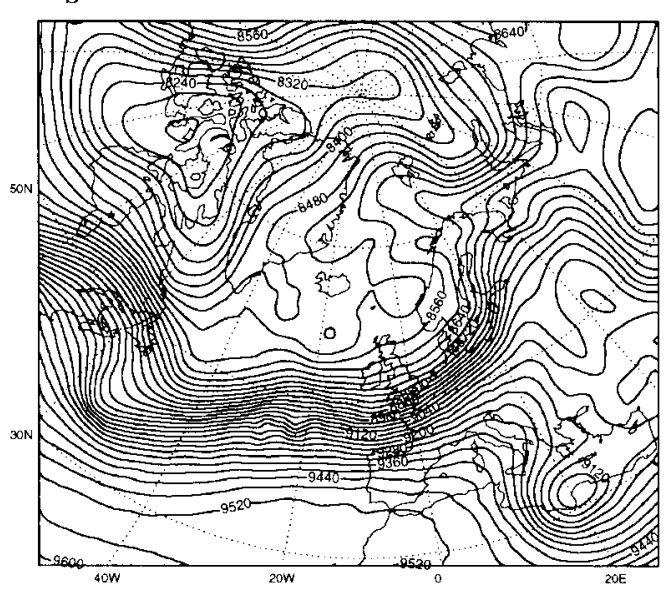

Fig. 8. Operational SMHI analysis for (a) $p_{\text {msl }}$ and (b) $300 \mathrm{hPa}$ height valid at 0000 UTC on 16 February 1995.

ity experiments (Figs. 10c, 11c) with the real forecast error field (Fig. 9a) at $+12 \mathrm{~h}$, we may conclude that the sensitivity difference field in the lateral boundary data experiment has a significantly better position than the sensitivity difference field in the initial data experiment. At the same time the results from the initial data experiment include sensitivity difference field structures which are not recovered by the boundary data experiment. We may thus conclude that the forecast errors for this particular case are likely to be related to initial as well as lateral boundary data errors. This is confirmed by running a sensitivity experiment with regard to both initial and lateral boundary data errors (see results for $+1 \mathrm{~h},+6 \mathrm{~h}$, $+12 \mathrm{~h},+18 \mathrm{~h}$ and $+24 \mathrm{~h}$ in Fig. 12). This experiment was carried out with a time interval of $\pm 1 \mathrm{~h}$ for the averaging of the lateral boundary perturbations, with a scaling coefficient of $\alpha_{\text {bound }}=5.0$ for the lateral boundary perturbations and with a scaling coefficient of $\alpha=0.1$ for the initial data perturbations. The sensitivity difference field pattern in Fig. 12c at $+12 \mathrm{~h}$ results in a sharper upper air trough over eastern Canada, which agrees very well with the forecast error pattern in Fig. 9a. We may also notice that the retrieved forecast error pattern at $+1 \mathrm{~h}$ reflects the initial data perturbation (see Fig. 10a) as well as the lateral boundary data perturbation at the initial time (Fig. 11a).

The sensitivity forecast experiment utilizing initial data perturbations and lateral boundary data perturbations during the first $12 \mathrm{~h}$ of the model integration was continued up to $+24 \mathrm{~h}$, using the original lateral boundary conditions between $+12 \mathrm{~h}$ and $+24 \mathrm{~h}$. The results of this experiment are included for $+18 \mathrm{~h}$ and $+24 \mathrm{~h}$ in Figs. 12d, $\mathrm{e}$, respectively, in the form of $300 \mathrm{hPa}$ wind and geopotential differences to the reference forecast, carried out without any initial and lateral boundary data perturbations. It is obvious that also the $+24 \mathrm{~h}$ forecast is significantly improved in the critical area south of Greenland due to the initial and boundary data perturbations provided by the +12 h sensitivity experiment.

The sensitivity of the boundary influence includes small amplitude artificial gravity waves in the boundary relaxation zone and a more balanced circulation just inside the inner boundary. Two further sensitivity experiments were carried out to check the impact of the time averaging period, introduced to reduce gravity wave oscillations in the lateral boundary perturbations. One experiment was done with a very short averaging period of \pm 10 minutes (see results at $+12 \mathrm{~h}$ in Fig. 13), and one experiment with a very long averaging period of $\pm 3 \mathrm{~h}$ (see results at $+12 \mathrm{~h}$ in Fig. 14). We notice that the experiment with the very short averaging period includes small scale (noisy) structures in the boundary relaxation zone, most likely associated with gravity wave oscillations added by unbalanced lateral boundary perturbations. The results from the experiment with the very long averaging period are smoother than the results from the standard experiment with an 

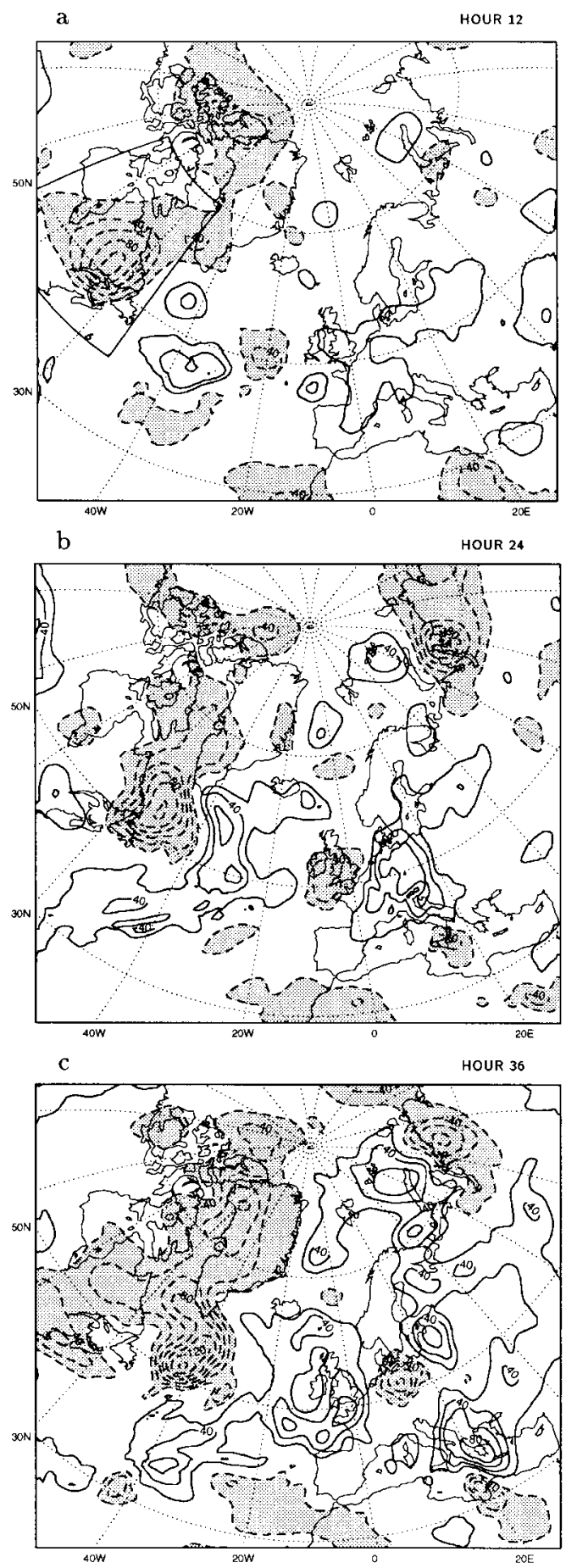

Tellus 50A (1998), 2 averaging period of $\pm 1 \mathrm{~h}$. Certain details in the sensitivity difference field pattern, as compared to the real forecast error pattern in Fig. 9a, are lost due to this smoothing effect, and we may conclude that $\pm 1 \mathrm{~h}$ seems to be a reasonable choice of averaging period for an efficient suppression of undesirable high frequency gravity wave oscillations from the lateral boundary perturbations produced by the adjoint model integration.

\section{Conclusions}

Operational HIRLAM forecasts from the Swedish Meteorological and Hydrological Institute (SMHI) for February 1995 were verified by means of rms and bias for the mean sea level pressure and $300-500 \mathrm{hPa}$ potential vorticity forecast errors. The time-series of these verification scores for February 1995 are utilized to identify two particularly poor forecast cases. An attempt to investigate the origin of these forecast failures is carried out by means of forecast sensitivity experiments based on the adjoint model technique. Sensitivity with respect to errors in initial as well as lateral boundary data are taken into account. It turns out that errors in initial data are the most likely explanations for one of the forecast failures (case I), while errors in initial as well as lateral boundary data may explain the second forecast failure (case b). The present study can be considered an extension of previous forecast sensitivity studies by Errico et al. (1993), Rabier et al. (1996) and Gustafsson and Huang (1996), since errors in initial as well as in lateral boundary data are taken into account in a systematic way.

Two different techniques for deriving lateral boundary perturbations to be applied in forecast sensitivity experiments were compared in this study. For the particular case investigated, it turned out to be advantageous to derive individual

Fig. 9. $300 \mathrm{hPa}$ height error fields (verification analysis - forecasts from operational SMHI HIRLAM) for forecast lengths of $12 \mathrm{~h}, 24 \mathrm{~h}$ and $36 \mathrm{~h}$ from 0000 UTC on 16 February 1995. Positive (negative) values are represented by solid (dashed) contours. Contours every $20 \mathrm{~m}$. The shaded areas correspond to errors $\leqslant-20 \mathrm{~m}$. The sector-formed area in (a) is used for calculation of the area-concentrated $12 \mathrm{~h}$ forecast error norm. 

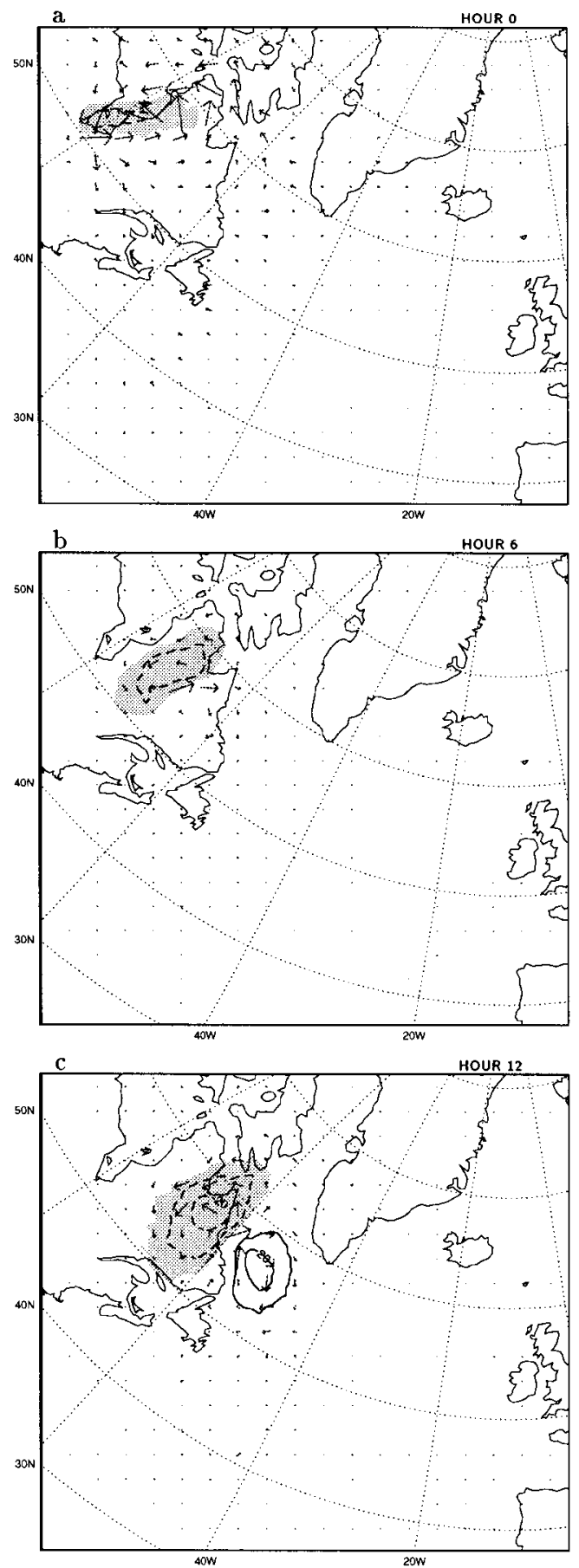

lateral boundary perturbations for each timestep of the forward sensitivity forecast model integration. This means that the linear time-interpolation of lateral boundaries is not included in the adjoint of the lateral boundary relaxation scheme. This result indicates that the applied 6-h interval for updating the lateral boundary conditions is too long, since important non-linear time variations of the boundary condition perturbations occur over such a long period. We have also shown, that it is advantageous to apply a time filtering to the calculated lateral boundary perturbations in order to suppress artificial high-frequency gravity wave oscillations originating from these lateral boundary perturbations for each timestep of the model integration. Furthermore, the general weakness of the Davies (1983) lateral boundary relaxation scheme, in that it does not distinguish between inflow and outflow regions, is a serious limitation for the performance of the adjoint of the boundary relaxation scheme. Regions of physical inflow become regions of forecast error gradient information outflow during the adjoint model integration, and this means that a straightforward application of boundary relaxation does not permit derived lateral boundary perturbations to extend all the way to the outermost gridpoints of the integration area. Artificial strong gradients are therefore created in the boundary relaxation zone. This may lead to spurious generation of unwanted gravity wave noise.

The successful application of the adjoint of the lateral boundary treatment for forecast sensitivity experiments, described in this paper, can simply be interpreted to mean that we are using observed information inside the model integration area to improve poor lateral boundary conditions. This result may be directly applied in four dimensional

Fig. 10. $300 \mathrm{hPa}$ height and wind differences between the sensitivity and reference $0 \mathrm{~h}, 6 \mathrm{~h}$ and $12 \mathrm{~h}$ forecasts from 0000 UTC on 16 February 1995 using initial perturbations only with $\alpha=0.1$ and the area-concentrated $12 \mathrm{~h}$ forecast error norm (Fig. 9a). Positive (negative) values are represented by solid (dashed) contours. Contours every $10 \mathrm{~m}$. The shaded areas correspond to retrieved forecast errors $\leqslant-5 \mathrm{~m}$. (a) $2 \mathrm{~m} / \mathrm{s}=8 \mathrm{~mm}$, (b and c) $20 \mathrm{~m} / \mathrm{s}=8 \mathrm{~mm}$. The western boundary of the model integration area coincides with the left side of the maps. 

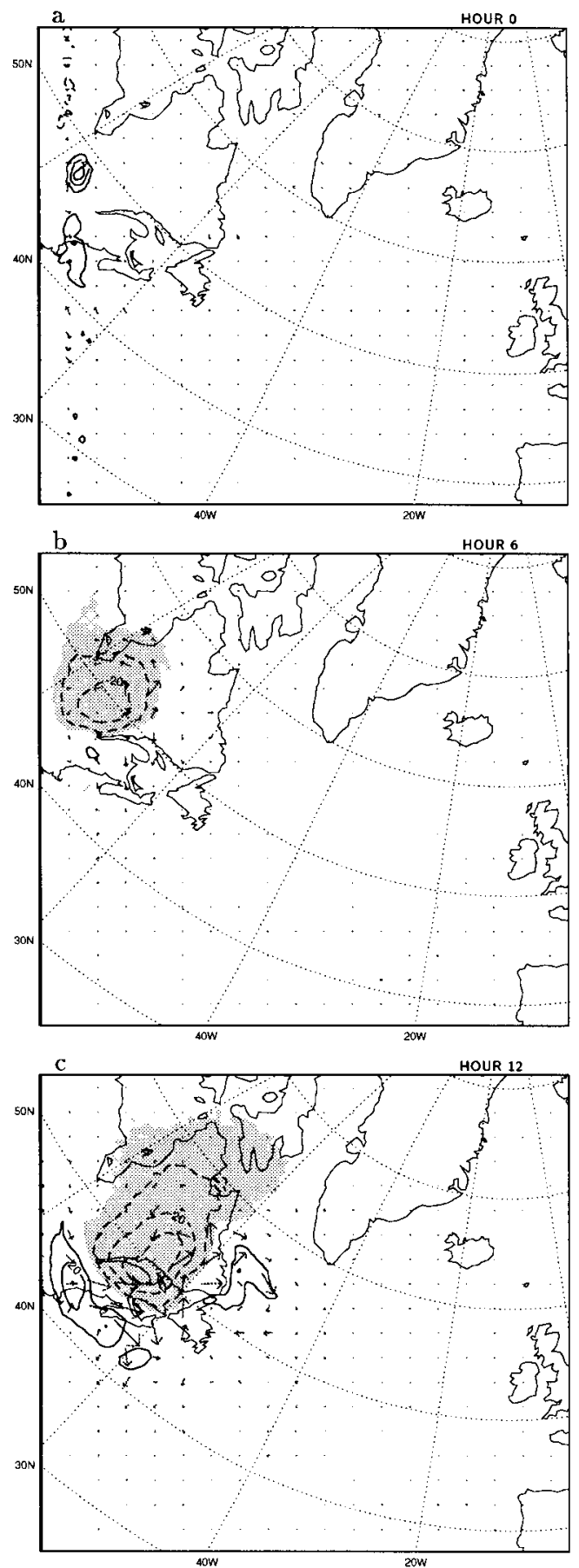

variational data assimilation (4DVAR); during the assimilation period the boundary values can be modified together with modifications in the initial state. 4DVAR assimilation of rainfall observations, including a similar control of lateral boundary conditions, has been tested by Zou and Kuo (1996). It remains to be seen how important this extension of 4DVAR for a limited area model may be in an operational setup. At present there is an intense effort in the HIRLAM project to construct a full 4DVAR system. A preliminary hybrid setup of this system has been tested by Huang et al. (1997). In this hybrid system the conventional optimum interpolation (OI) analysis procedure is augmented by a sensitivity type of integration. The first guess field is improved by projecting the difference between the preliminary OI analysis and first guess field back to the initial state of the first guess integration. A new first guess integration is then carried out with a perturbed initial state. This perturbation could also be applied to the boundary values during the first guess integration period, as demonstrated in this study.

In practice the boundary values used during data assimilation with a limited area model can be very old compared to the forecast integration time of the limited area model. The global model used to produce the boundary values can be based on an initial state which is 36 hours older than the most recent observational information, which is fed into the limited area data assimilation. In this study we have shown how the adjustment of boundary data during the first twelve hours of a forecast can improve the subsequent 36 hours of forecast time. We feel that this gives a strong motivation for including a procedure of the type discussed here in a limited area model data assimilation system. Our results reinforce the conclusions reached by Gustafsson (1990) and furthermore we have seen very clear evidence that an improved mathematical formulation of the lateral boundary condition is needed in limited area models of the HIRLAM type.

Fig. 11. As in Fig. 10, but using only the lateral boundary perturbations for the sensitivity forecast run with $\alpha_{\mathrm{bound}}=5.0$ and with a time-averaging period of $\pm 1 \mathrm{~h}$ : (a) $0 \mathrm{~h}$ (boundary perturbation), (b) $6 \mathrm{~h}$, (c) $12 \mathrm{~h}$. (a) Contour interval of $2 \mathrm{~m}, 1 \mathrm{~m} / \mathrm{s}=8 \mathrm{~mm},(\mathrm{~b}, \mathrm{c})$ contour interval of $10 \mathrm{~m}, 20 \mathrm{~m} / \mathrm{s}=8 \mathrm{~mm}$.

Tellus 50A (1998), 2 

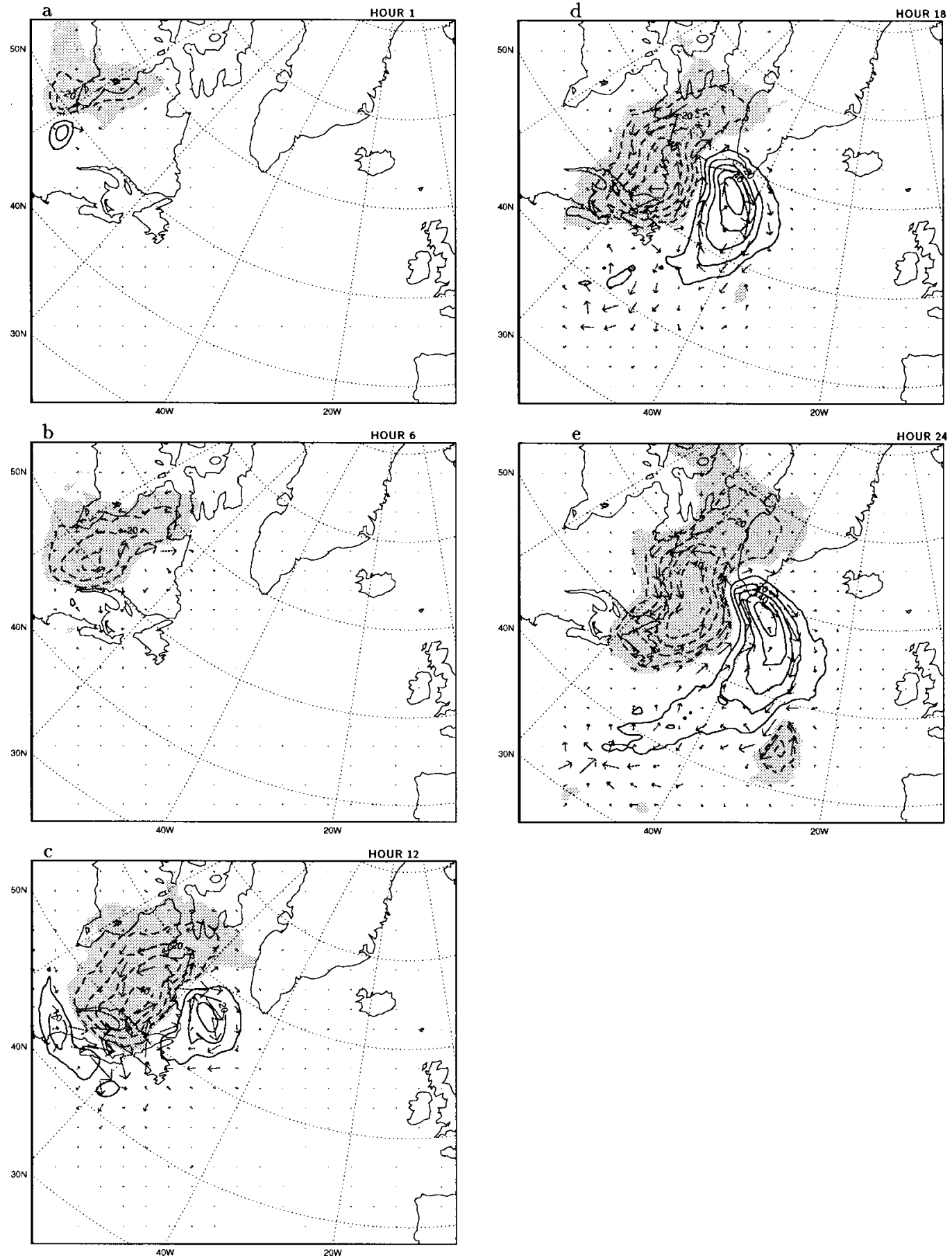

Fig. 12. As in Fig. 10, but using initial as well as boundary perturbations for the sensitivity forecast run with $\alpha=$ 0.1 and $\alpha_{\text {bound }}=5.0$ and with a time averaging period of $\pm 1 \mathrm{~h}$ : (a) $1 \mathrm{~h}$, (b) $6 \mathrm{~h}$, (c) $12 \mathrm{~h}$, (d) $18 \mathrm{~h}$, (e) $24 \mathrm{~h} .20 \mathrm{~m} / \mathrm{s}=8 \mathrm{~mm}$. 


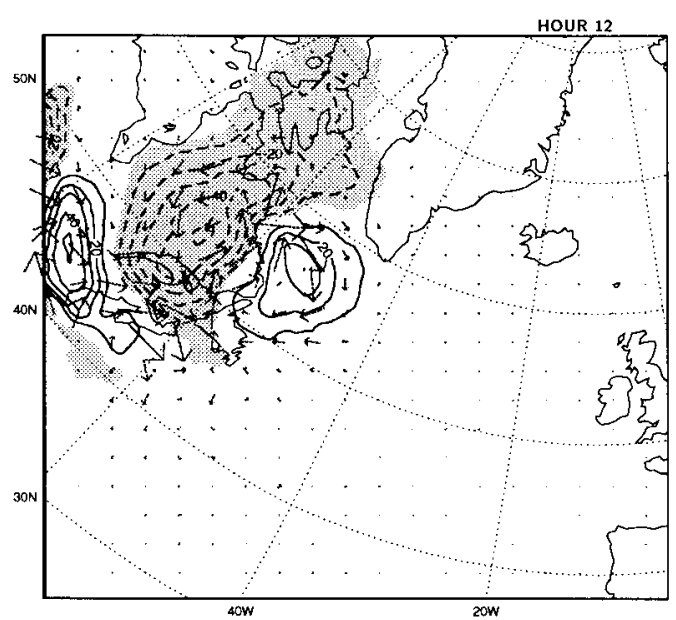

Fig. 13. As in Fig. 10c, $12 \mathrm{~h}$ only, but using initial as well as boundary perturbations for the forecast sensitivity run with $\alpha=0.1$ and $\alpha_{\text {bound }}=5.0$ and a time-averaging period of $\pm 10 \mathrm{~min} .20 \mathrm{~m} / \mathrm{s}=8 \mathrm{~mm}$.

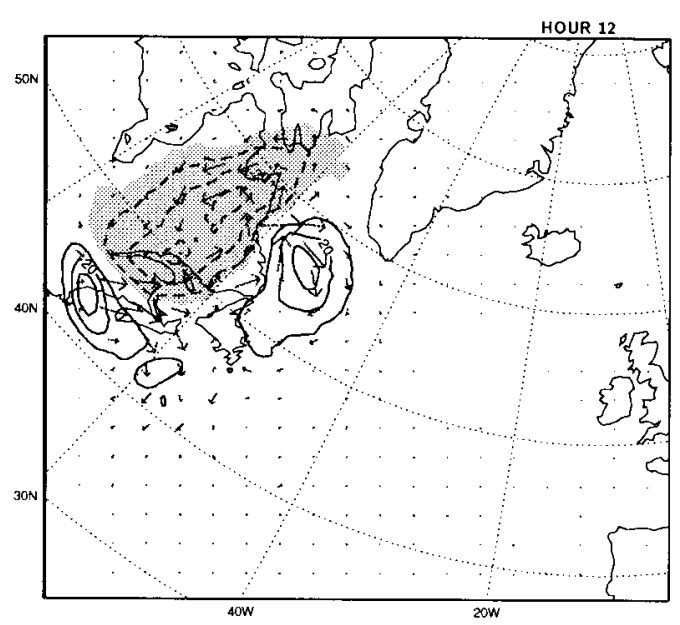

Fig. 14. As in Fig. 10c, $+12 \mathrm{~h}$ only, but using initial as well as boundary perturbations for the forecast sensitivity run with $\alpha=0.1$ and $\alpha_{\text {bound }}=5.0$ and a time-averaging period of $\pm 3 \mathrm{~h} .20 \mathrm{~m} / \mathrm{s}=8 \mathrm{~mm}$.

\section{Acknowledgements}

We would like to thank Dr. X.-Y. Huang from the Danish Meteorological Institute, who contributed to the development of the HIRLAM system for forecast sensitivity experiments and to discussions of the present work. Two anonymous reviewers contributed with suggestions and ques- tions that helped to improve this paper. This research was supported by the Icelandic Council of Science.

\section{Appendix}

Advection properties of the boundary zone relaxation scheme

To demonstrate the propagation properties of the boundary zone scheme we have found that the following very simple model system can be used. The results are similar to what Davies (1983) already has shown, but they give additional insight into the specific properties of the scheme which are examined in this paper.

Consider a linear advection equation where $A(x, t)$ is linearly advected with a $U>0$. Assume a semi-Lagrangian time stepping scheme with a time step such that the CFL number is equal to one,

$\Delta t=\frac{\Delta x}{U}$.

This implies that

$A(x, t+\Delta t)=A(x-\Delta x, t)$.

If we now apply a relaxation boundary zone to this problem we may write

$$
\begin{aligned}
\mathrm{A}(x, t+\Delta t)= & g(x) A_{\mathrm{b}}(x, t+\Delta t) \\
& +(1-g(x)) A_{\mathrm{i}}(x, t+\Delta t),
\end{aligned}
$$

where $A_{\mathrm{b}}$ is the externally prescribed boundary forcing field and $A_{\mathrm{i}}$ is the interior domain field. The boundary zone relaxation function is given by $g(x)$ and we assume the outermost boundary point to be located at $x=0$. Let us now determine the relative weights of the different external boundary point values at the first fully interior point of the computational domain, i.e., the first point where $g(x)=0$. Let us call this point $x_{k}$. A successive insertion of (A.2) into (A.3) gives:

$$
\begin{aligned}
& A\left(x_{\mathrm{k}}, k \Delta t\right) \\
&= A\left(x_{k}-\Delta x,(k-1) \Delta t\right) \\
&= g\left(x_{k}-\Delta x\right) A_{\mathrm{b}}\left(x_{k}-\Delta x,(k-1) \Delta t\right) \\
&+\left(1-g\left(x_{k}-\Delta x\right)\right) A_{\mathrm{i}}\left(x_{k}-\Delta x,(k-1) \Delta t\right) \\
&= g\left(x_{k}-\Delta x\right) A_{\mathrm{b}}\left(x_{k}-\Delta x,(k-1) \Delta t\right) \\
&+\left(1-g\left(x_{k}-\Delta x\right)\right) A\left(x_{k}-2 \Delta x,(k-2) \Delta t\right),
\end{aligned}
$$


and so on until we finally get

$$
\begin{aligned}
& \mathrm{A}\left(x_{k}, k \Delta t\right) \\
& =g\left(x_{k}-\Delta x\right) A_{\mathrm{b}}\left(x_{k}-\Delta x,(k-1) \Delta t\right) \\
& \quad+\left(1-g\left(x_{k}-\Delta x\right)\right) g\left(x_{k}-2 \Delta x\right) \\
& \quad \times A_{\mathrm{b}}\left(x_{k}-2 \Delta x,(k-2) \Delta t\right) \\
& \quad+\left(1-g\left(x_{k}-\Delta x\right)\right)\left(1-g\left(x_{k}-2 \Delta x\right) g\left(x_{k}-3 \Delta x\right)\right. \\
& \quad \times A_{\mathrm{b}}\left(x_{k}-3 \Delta x,(k-3) \Delta t\right)+\ldots+ \\
& \quad+\left(1-g\left(x_{k}-\Delta x\right)\right)\left(1-g\left(x_{k}-2 \Delta x\right)\right. \\
& \quad \times \ldots g(0) A_{\mathrm{b}}(0,0) .
\end{aligned}
$$

The relative weight of each externally prescribed boundary point thus depends on the functional form of $g(x)$. Let us assume a four point boundary zone where $g(0)=1 ; g(\Delta x)=0.9 ; g(2 \Delta x)=0.5$; and $g(3 \Delta x)=0.1$. From (A.5) we obtain

$$
\begin{aligned}
\mathrm{A}(4 \Delta x, 4 \Delta t) \\
=0.1 A_{\mathrm{b}}(3 \Delta x, 3 \Delta t)+0.45 A_{\mathrm{b}}(2 \Delta x, 2 \Delta t) \\
\quad+0.405 A_{\mathrm{b}}(\Delta x, \Delta t),+0.045 A_{\mathrm{b}}(0,0),
\end{aligned}
$$

thus showing that at the time when the information from the outermost boundary point has reached the interior of the domain very little of the original amplitude remains. Most of the information at the first fully interior point comes from boundary value information at the second and third boundary zone points. This statement may be generalized to other boundary zone functions. The weight of the outer-most boundary point is always a product of several weighting factors significantly less than one and thus will be a small number, while the information coming from the boundary zone point adjacent to the first fully interior point must also be small as the weighting factor must be near zero at this point.

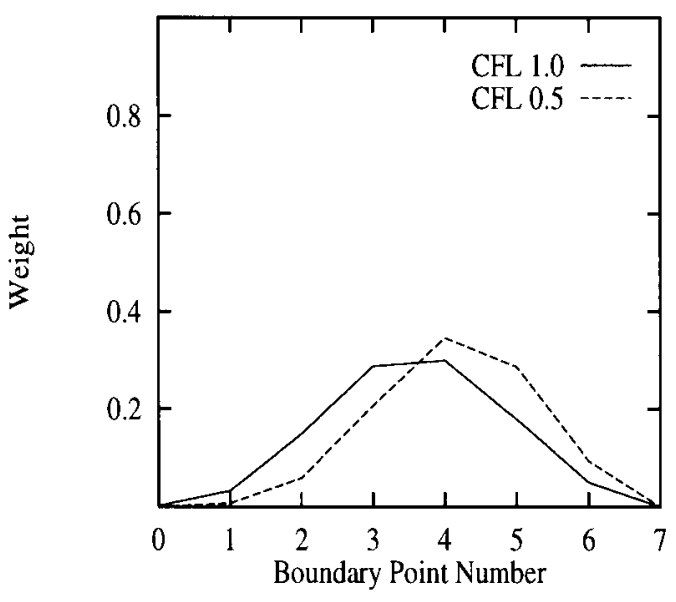

Fig. 15. Efficient weights of lateral boundary values as determined by linear advection over the entire boundary relaxation zone to form the model value just inside this zone. Fast advection ( $\mathrm{CFL}=1$, full line) and slow advection $(\mathrm{CFL}=0.5$, dashed line).

Larger values occur at intermediate boundary zone points.

The boundary zone relaxation function used in this study is cosine-shaped and the width of the boundary relaxation zone is 8 gridpoints. A similar advection scheme as in the demonstration above, extended to CFL numbers also smaller than one, was applied to obtain the weights given to the externally prescribed boundary points in the solution just inside the boundary relaxation zone. These sensitivity weights obtained for CFL numbers 1.0 and 0.5 are illustrated in Fig. 15. The general conclusion reached with the help of equation (A.6) still holds, most of the information comes from the middle of the boundary relaxation zone. It can also be seen that the area of sensitivity is shifted towards the inner integration area for smaller CFL numbers.

\section{REFERENCES}

Davies, H. C. 1983. Limitations of some common lateral boundary schemes used in NWP models. Mon. Wea. Rev. 111, 1002-1012.

Eliassen, A. and Thorsteinsson, S. 1984. Numerical studies of stratified air flow over a mountain ridge on the rotating earth. Tellus 36A, 172-186.

Errico, R. M., Vukićević, T. and Raeder, K. 1993. Comparison of initial and lateral boundary condition sensitivity for a limited-area model. Tellus 45A, 539-557.

Gustafsson, N. 1990. Sensitivity of limited area model data assimilation to lateral boundary condition fields. Tellus 42A, 109-115.

Gustafsson, N. 1991. The HIRLAM model. Proceedings of the ECMWF Seminar on Numerical methods in atmospheric models, 9-13 September, 1991; available from the European Centre for Medium Range Weather Forecasting, Shinfield Park, Reading, England.

Gustafsson, N. and Huang, X.-Y. 1996. Sensitivity experiments with the spectral HIRLAM and its adjoint. Tellus 48A, 501-517. 
Gustafsson, N. and McDonald, A. 1996. A comparison of the HIRLAM grid point and spectral semiLagrangian models. Mon. Wea. Rev. 124, 2008-2022.

Gustafsson, N., Lönnberg, P. and Pailleux, J. 1997. Data assimilation for high resolution limited area models. J. Met. Soc. Japan 75B, 367-382.

Huang, X.-Y., Gustafsson, N. and Källén, E. 1997. Using an adjoint model to improve an optimum interpolation based data assimilation system. Tellus 49A $161-176$.

Källén, E. and Huang, X.-Y. 1988. The influence of isolated observations on short-range numerical weather forecasts. Tellus 40A, 324-336.

Källén, E. 1995. The HIRLAM 3 project. In: Modern dynamical meteorology, Proceedings from a Symposium in honour of Prof. Aksel Wiin-Nielsen, University of Copenhagen, 1995, pp. 15-20.
Le Dimet, F.-X. and Talagrand, O. 1986. Variational algorithms for analysis and assimilation of meteorological observations. Tellus 38A, 97-110.

Orlanski, I. 1976. A simple boundary condition for unbounded hyperbolic flows. J. Comp. Phys. 21, 251-269.

Rabier, F., Klinker, E., Courtier, P. and Hollingsworth, A. 1996. Sensitivity of two-day forecast errors over the Northern Hemisphere to initial conditions. Q. J. R. Meteorol. Soc. 121, 121-150.

Thorsteinsson, S. 1988. Finite amplitude stratified air flow past isolated mountains on an f-plane. Tellus 40A, 220-236.

Zou, X. and Kuo, Y.-H. 1996. Rainfall assimilation through an optimal control of initial and boundary conditions in a limited-area mesoscale model. Mon. Wea. Rev. 124, 2859-2882. 